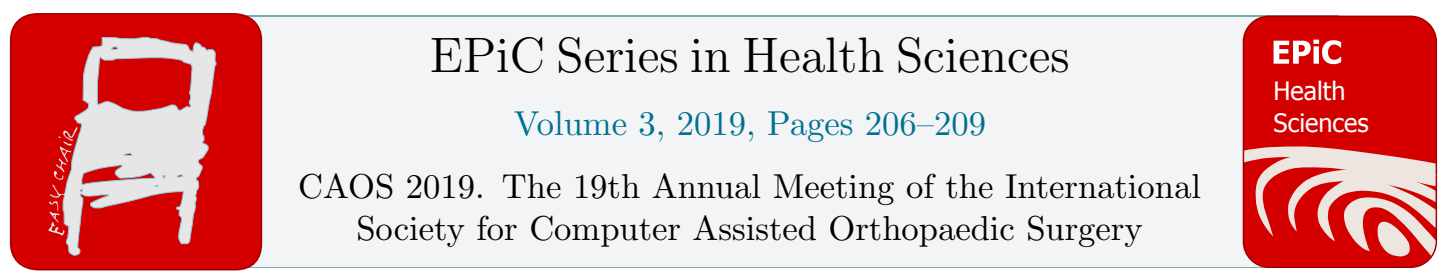

\title{
Robotic-assisted total hip arthroplasty yields enhanced surgical and clinical outcomes compared to manual hip arthroplasty using the same implant system
}

\author{
Seth A. Jerabek MD ${ }^{1}$, Russell Cohen $\mathrm{MD}^{2}$, Alana Levine ${ }^{3}$, \\ Alice $\mathrm{Cruz}^{3}$ and Laura Scholl ${ }^{3}$ \\ ${ }^{1}$ Hospital for Special Surgery, New York, NY \\ ${ }^{2}$ Tucson Orthopaedic Institute, Tucson, AZ \\ ${ }^{3}$ Stryker Orthopaedics, Mahwah, NJ
}

\begin{abstract}
In a prospective, non-randomized trial, 222 hips across seven centers received the same THA system of a tapered wedge stem and novel additively manufactured titanium porous clusterhole acetabular shell in primary THA. 113 hips were implanted with a robotic-assisted surgical system while 109 hips were implanted manually. The robotic-assisted cohort yielded a similar mean skin-to-skin surgical time of 80.35 minutes compared to 79.35 minutes for the manual cohort $(\mathrm{p}=0.8895)$, along with a shorter hospital stay of 0.96 days compared to 1.59 days $(\mathrm{p}<0.0001)$. The robotic-assisted cohort resulted in a lower blood loss of $199.8 \mathrm{cc}$ compared to $310.1 \mathrm{cc}$ for the manual cohort $(\mathrm{p}<0.0001)$. Standard deviation for incision length was $81 \%$ of the value in robotic-assisted cases when comparing with manual cases $(2.2 \mathrm{~cm}$ vs. $2.7 \mathrm{~cm})$. Robotic-assisted THA cases demonstrated improved clinical outcomes with a higher 6-week postoperative HHS (81.0 vs. 78.4), physical VR-12 (39.5 vs. 35.9), and LEAS (9.2 vs. 8.0). The EQ-5D treatment effect size at 6-weeks postoperative for robotic-assisted cases exceeded a large effect with a value of 0.91 , while the effect size for manual cases was 0.59 . Robotic-assisted 3-D patient specific planning and haptically guided robotic-assisted surgical execution demonstrated an improved operative experience relative to blood loss, and incision length. The robotic cohort also yielded improved early clinical and functional outcomes when comparted to manual techniques at six weeks. These early, enhanced robotic-assisted THA results support surgeons using robotics with the goals of decreased patient pain, greater range of motion, and increased joint stability.
\end{abstract}

\section{Introduction}

Several radiographic studies have demonstrated the precision and accuracy of component placement in robotic-assisted total hip arthroplasty1,2 (THA). However, no studies have evaluated the 
intraoperative experience and early clinical outcomes between robotic and manual techniques. This is the first study to compare intraoperative surgical information and early postoperative clinical outcomes between robotic-assisted and manual implantation using the same novel additively manufactured porous $^{3}$ acetabular shell and a tapered wedge stem.

\section{Methods}

In a prospective, non-randomized trial, 222 hips across seven centers received the same THA system of a tapered wedge stem (Accolade II, Stryker, Mahwah, NJ) and an additively manufactured titanium porous clusterhole acetabular shell (Trident II Tritanium, Stryker, Mahwah, NJ) in patients undergoing a primary THA. In our cohort, 113 hips were implanted with a robotic-assisted surgical system (Mako/Stryker, Fort Lauderdale, FL) by four different surgeons, while 109 hips were implanted manually by three different surgeons. Demographic and surgical information was collected, along with 6-week post-operative clinical outcomes including: Harris Hip Score (HHS), Lower Extremity Activity Scale (LEAS), EuroQol-5D (EQ-5D) and Veterans Rand 12 (VR12). There were no differences in body mass index (BMI), age, or ASA Score between the robotic-assisted and manual cohorts.

\section{Results}

The robotic-assisted cohort yielded a similar mean skin-to-skin surgical time of 80.35 minutes compared to 79.35 minutes for the manual cohort ( $\mathrm{p}=0.8895$ ), along with a shorter hospital stay of 0.96 days compared to 1.59 days $(\mathrm{p}<0.0001)$. The robotic-assisted cohort resulted in a lower blood loss of $199.8 \mathrm{cc}$ compared to $310.1 \mathrm{cc}$ for the manual cohort $(\mathrm{p}<0.0001)$. These trends translated to improved clinical outcomes with the robotic-assisted THA cases demonstrating a higher 6-week postoperative HHS (81.0 vs. 78.4), physical VR-12 (39.5 vs. 35.9), and LEAS (9.2 vs. 8.0). Robotic-assisted cases reported a similar preoperative pain specific to indoor and outdoor housework yet experienced a significantly lower postoperative pain compared to manual cases. (Figure 1). The EQ-5D treatment effect size at 6-weeks postoperative for robotic-assisted cases exceeded a large effect with a value of 0.91 , while the effect size for manual cases was 0.59 (Figure 2).

\section{Discussion}

Robotic-assisted 3-D patient specific planning and haptically guided robotic-assisted surgical execution demonstrated an improved operative experience relative to blood loss, and incision length. The decrease in blood loss may be contributed to the use of a single-ream technique for acetabular preparation. The robotic cohort also yielded improved early clinical and functional outcomes when comparted to manual techniques at six weeks. These early, enhanced robotic-assisted THA results support surgeons using robotics with the goals of decreased patient pain, greater range of motion, and increased joint stability. ${ }^{4,5}$ The ability to plan and recreate the desired hip center of rotation, hip length, and offset likely contribute to the improved clinical outcomes. ${ }^{6,7}$

Although many confounding variables were controlled for, longer term follow-up with larger numbers may be required to confirm enhanced outcomes and surgical experiences using the roboticassisted total hip arthroplasty. 


\section{Figures}

Figure 1. Percentage of patients experienced no pain or a little pain specific to indoor and outdoor housework in robotic versus manual cases.

Percentage of no pain or a little bit pain

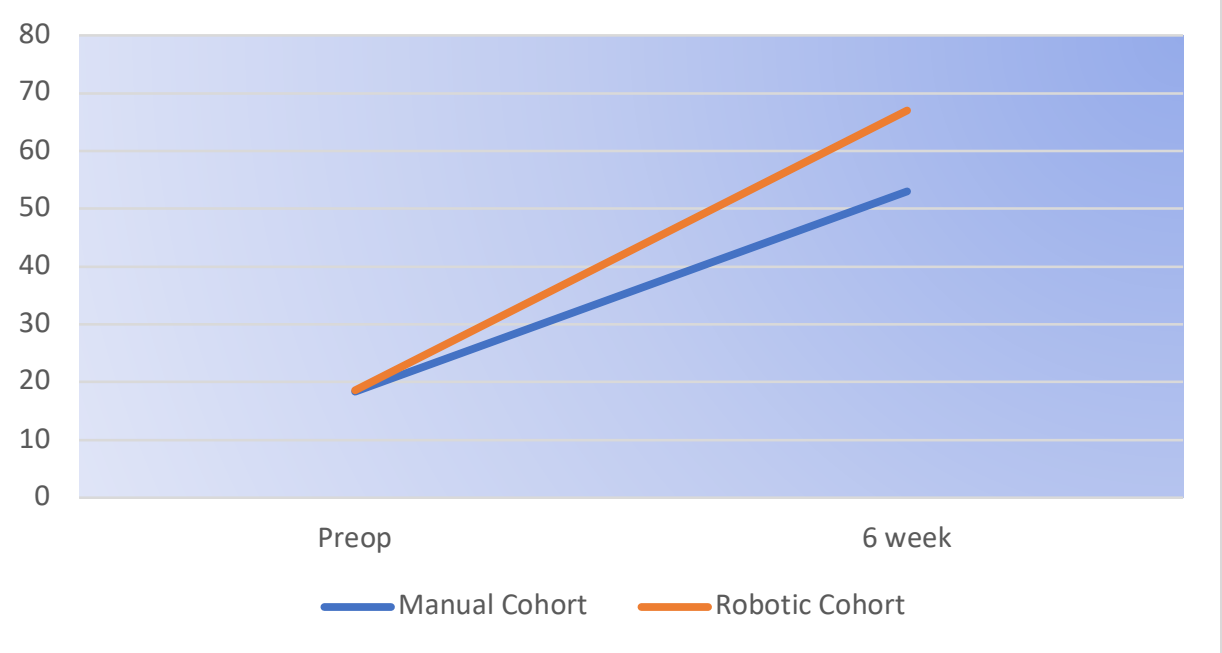

Figure 2. EQ5D effect size at 6-weeks postoperative for robotic and manual cases.

EQ5D effect size at 6 week

Small effect: 0.2

Medium effect: 0.5

Large effect: 0.8 


\section{References}

1. Nawabi DH; Conditt MA; Ranawat AS; Dunbar NJ; Jones, J; Banks S, Padgett DE. Haptically guided robotic technology in total hip arthroplasty - A cadaveric investigation. Journal of Engineering in Medicine. December 2012;227(3):302-309.

2. Domb BG, et al,. Does Robotic-Assisted Computer-Navigation Affect Acetabular Cup Positioning in Total Hip Arthroplasty in the Obese Patient? A Comparison Study. 14th Annual CAOS Meeting, June18-21, 2014, Milan, IT.

3. Stryker R\&D Technical Report: Characterizing the Physical Properties of the Trident II Tritanium Acetabular Shell. May 22, 2017. A0021722

4. Mirza S, Dunlop D G, Panesar S, Syed G N, Shafat G, Saif S. Basic Science Considerations in Primary Total Hip Replacement Arthroplasty. The Open Orthopaedics Journal. 2010;4, 169-180

5. Bukowkski BR, Anderson P, Khlopas A, Chugthai M, Mont MA, Illgen RL $2^{\text {nd }}$. Improved Functional Outcomes with Robotic Compared with Manual Total Hip Arthroplasty. Surg Technol Int. 2016 Oct 26;29:303-308.

6. Maloney, William, Keeney, James. Leg length discrepancy after total hip arthroplasty. J Arhtroplasty. 2004 Jun; 19(4 Suppl 1):108-10.

7. Nodzo SR, Chang CC, Carroll KM, Barlow BT, Banks SA, Padgett DE, Mayman DJ, Jerabek SA. Intraoperative placement of total hip arthroplasty components with robotic-arm assisted technology correlates with postoperative implant position: a CT-based study. Bone Joint J. 2018 Oct;100-B (10):1303-1309. 